\title{
Spatial Terahertz Modulator
}

\section{SUBJECT AREAS:}

TERAHERTZ OPTICS

OPTICAL PHYSICS

Received

1 November 2013

Accepted

6 November 2013

Published

26 November 2013

Correspondence and requests for materials should be addressed to

Y.Z. (yzhang@mail. cnu.edu.cn)

* These authors contributed equally to this work.

\author{
Zhenwei Xie ${ }^{1,2 *}$, Xinke Wang ${ }^{1 *}$, Jiasheng Ye', Shengfei Feng ${ }^{1}$, Wenfeng Sun', Tahsin Akalin ${ }^{3}$ \\ \& Yan Zhang ${ }^{1,2}$
}

\begin{abstract}
'Department of Physics, Capital Normal University, Beijing Key Lab for Terahertz Spectroscopy and Imaging, and Key Laboratory of Terahertz Optoelectronics, Ministry of Education, Beijing 100048, China, ${ }^{2}$ Department of Physics, Harbin Institute of Technology, Harbin 150001, China, ${ }^{3}$ IEMN, Lille 1 University, 59652, Villeneuve d Ascq, France.
\end{abstract}

Terahertz (THz) technology is a developing and promising candidate for biological imaging, security inspection and communications, due to the low photon energy, the high transparency and the broad band properties of the $\mathrm{THz}$ radiation ${ }^{1-3}$. However, a major encountered bottleneck is lack of efficient devices to manipulate the $\mathrm{THz}$ wave, especially to modulate the $\mathrm{THz}$ wave front. A wave front modulator should allow the optical or electrical control of the spatial transmission (or reflection) of an input $\mathrm{THz}$ wave and hence the ability to encode the information in a wave front ${ }^{4}$. Here we propose a spatial THz modulator (STM) to dynamically control the $\mathrm{THz}$ wave front with photo-generated carriers. A computer generated $\mathrm{THz}$ hologram is projected onto a silicon wafer by a conventional spatial light modulator (SLM). The corresponding photo-generated carrier spatial distribution will be induced, which forms an amplitude hologram to modulate the wave front of the input $\mathrm{THz}$ beam. Some special intensity patterns and vortex beams are generated by using this method. This all-optical controllable STM is structure free, high resolution and broadband. It is expected to be widely used in future $\mathrm{THz}$ imaging and communication systems.

andwiched between the microwave and infrared, $\mathrm{THz}$ radiation has been notoriously difficult to produce, modulate and detect ${ }^{1-3}$. Recent progresses such as quantum-cascade lasers ${ }^{5,6}$, terahertz wave generation through a nonlinear $\mathrm{crystal}^{7}$ and $\mathrm{THz}$ time-domain spectroscopy ${ }^{8}$ are promoting this subject into one of the most rapidly growing fields. High performance devices to control and manipulate the $\mathrm{THz}$ radiation are in urgent demand to develop sophisticated imaging and communication system. The filters, absorbers and polarizers based on graphene, frequency selective surface, metamaterials and photonic crystals have been reported ${ }^{9-18}$. However, the wave front modulation devices are still lacking. Recently, a novel technology based on the metasurface has been demonstrated to generate the desired wave front distribution ${ }^{19,20}$. Unfortunately, the specific function of this kind of devices has been determined at the moment of their design and could not be flexibly changed any more. The SLM which has been widely used in the visible light band can optically or electrically control the spatial transmission (or reflection) of an input light beam and encode information in the wave front ${ }^{4}$. The SLM always plays an important role in optical information processing, three dimensional image display, optical interconnections and real-time beam shaping. Usually, the SLM is realized through liquid crystals, magneto-optic materials or deformable mirrors. However, such mechanisms cannot work well in the THz regime due to the lack of suitable materials and the size mismatching between the micro-machined components and the $\mathrm{THz}$ wavelength ${ }^{21}$. In order to obtain the STM, a novel technology needs to be explored.

The STM requires an array of small building blocks that can independently control the transmission (or reflection) of a $\mathrm{THz}$ beam at different positions. Chan et. al. proposed a multi-pixel spatial modulator for $\mathrm{THz}$ beams using active $\mathrm{THz}$ metamaterials ${ }^{22}$. The transmission of each unit cell can be independently controlled by applying a small external voltage at room temperature. Even the fabrication process is complex, the spatial resolution is low and the response band is narrow, there is still no alternative method so far.

Here we propose an all-optical controllable STM, which is structure free, high resolution and broadband, to actively manipulate the THz wave front. This STM paves an avenue for $\mathrm{THz}$ imaging, $\mathrm{THz}$ information processing and $\mathrm{THz}$ communications. In this letter, we use this STM to produce special $\mathrm{THz}$ beams based on the computer generated holography.

The prototype of this STM is shown in Fig. 1a. The control beam modulated by a conventional SLM illuminates the surface of a semiconductor wafer to produce the corresponding pattern of photo-generated carriers. The transmission of the $\mathrm{THz}$ beam is governed by the density of carriers. Hence, the transparency at different positions of the semiconductor wafer can be independently modulated. If the pattern projected on the semiconductor wafer is a THz computer generated hologram $(\mathrm{CGH})$, the diffracted $\mathrm{THz}$ wave will generate the preset wave front with a special phase and amplitude distribution, for example, a THz vortex beam. 


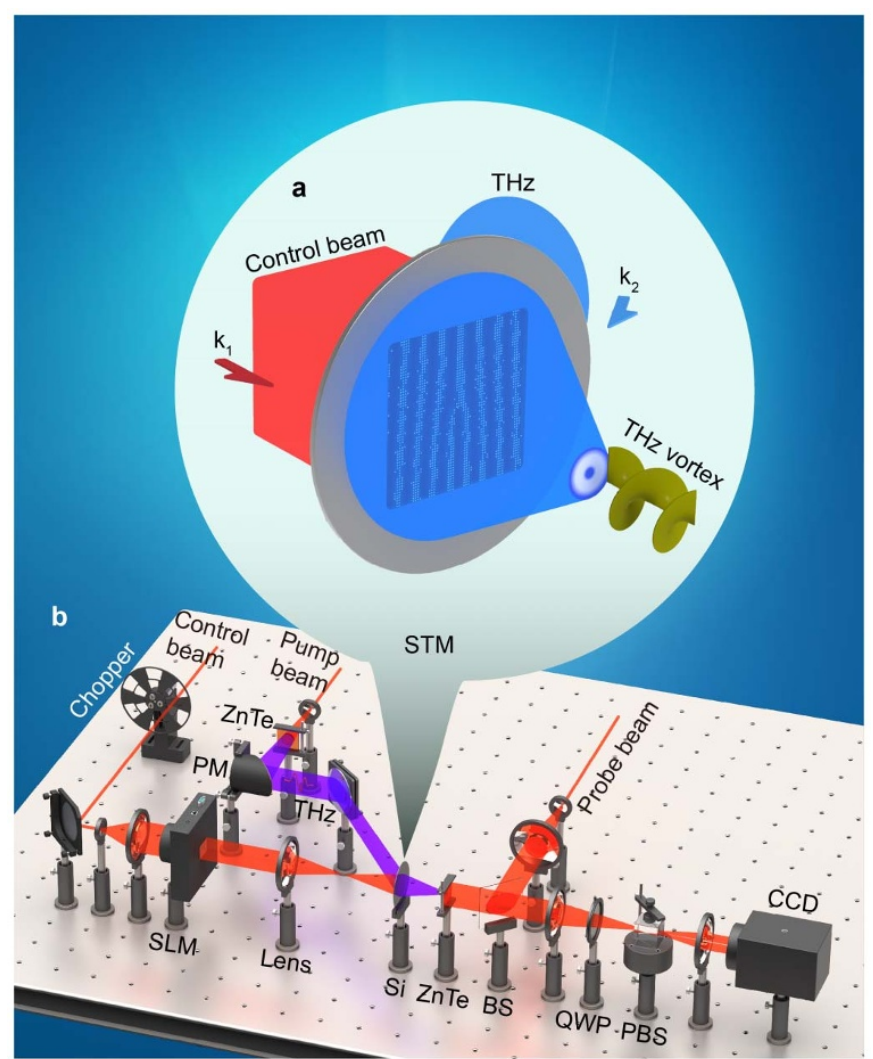

Figure $1 \mid$ Prototype and experimental configuration of the spatial terahertz modulator (STM). (a), Prototype of the STM. The control beam with an intensity pattern illuminates a semiconductor wafer, so that the localized photo-induced carriers are generated to modulate the transmitted $\mathrm{THz}$ wave front. The diffracted $\mathrm{THz}$ radiation will produce the designed special beam, such as a THz vortex. (b), Experimental configuration of the STM.

An infrared pump $\mathrm{THz}$ imaging system, which is developed from our previous $\mathrm{THz}$ focal plane imaging system ${ }^{23,24}$, is used to demonstrate this STM. As shown in Fig. 1b, 100 femtosecond pulses with a $800 \mathrm{~nm}$ central wavelength from a Ti: sapphire regenerator amplifier are split into three beams. These three beams are control beam, pump beam and probe beam, respectively. The control beam is utilized to construct holograms on a $500 \mu \mathrm{m}$ thick silicon wafer, the pump beam to generate the $\mathrm{THz}$ radiation using a $\mathrm{ZnTe}$ crystal and the probe beam to detect the two-dimensional THz information.

To characterize the modulation depth of this STM, the relative transmission $T$ of the $1.0 \mathrm{THz}$ wave through the silicon wafer under different intensities of the control beam are measured and displayed in Fig. 2a. The relative transmission is defined as $T=E / E_{0}$, where $E_{0}$ and $E$ are the transmitted $\mathrm{THz}$ amplitudes without and with the control beam, respectively. It can be found that the relative transmission of the $\mathrm{THz}$ wave is reduced quickly with the increase of the control beam intensity. The modulation depth can arrive 98.6\% when the control beam intensity is $543.2 \mu \mathrm{J} / \mathrm{cm}^{2}$. A good linear relationship between the relative transmission and the control beam intensity is observed within the range from 0 to $100 \mu \mathrm{J} / \mathrm{cm}^{2}$. Compared with the electrically controlled $\mathrm{THz}$ spatial modulator ${ }^{22}$, this optically controlled STM has higher resolution and better modulation depth. To evaluate the resolving power of the STM, a 1951 USAF 1X resolution test chart (Fig. 2b) is inserted into the control beam and projected onto the silicon wafer. The $\mathrm{THz}$ images of elements 3 to 6 in group 1 at $1.0 \mathrm{THz}, 1.3 \mathrm{THz}$, and $1.6 \mathrm{THz}$ are shown in Fig. 2c-e, respectively. The elements 3 and 4 in Fig. $2 c$ and all the elements in Fig. 2d-e can be clearly distinguished, which indicates that the STM can achieve resolution better than $140 \mu \mathrm{m}$. At the same time, the broadband property of the STM is shown in Fig. 2c-e, which originates from the intrinsic broadband response of photogenerated carriers.

As a typical application, the STM is used to display THz CGHs for generating intensity patterns. The control beam is normally incident onto the silicon wafer. A spatial light modulator (Holoeye LC2002, resolution $800 \times 600$, pixel size $32 \mu \mathrm{m}$ ) is inserted into the control beam to excite the carrier distribution of the designed THz CGHs. In order to achieve better results, an off-line holography configuration is adopted. The THz radiation is reflected onto the silicon wafer with an incident angle of 37 degrees. The transmission of the THz CGH can be expressed as

$$
T_{C G H}=C+2 O_{T H z} R_{T H z} \cos \Delta \varphi,
$$

where $C$ is a constant to ensure that the transmission is positive, $O_{T H z}$ and $R_{T H z}$ are the amplitude distributions of the demanded and reference $\mathrm{THz}$ wave fronts, respectively. $\Delta \varphi$ is the phase difference between $O_{T H z}$ and $R_{T H z}$.

Different intensity distributions are generated by using this method. Three letters " $C$ ", "N" and " $U$ " are required to be shown on the plane which is $2 \mathrm{~cm}$ away from the silicon wafer. The size of each letter is about $4 \mathrm{~mm} \times 6 \mathrm{~mm}$. The calculated CGHs shown in Fig. $3 \mathrm{a}-\mathrm{c}$ are displayed on the optical SLM and projected onto the surface of the silicon wafer, respectively. The complex amplitude distribution of the $\mathrm{THz}$ beam on the observation plane is captured with the $\mathrm{THz}$ focal plane imaging system. The intensity distributions are presented in Fig. 3d-f, respectively. It can be seen that the three letters " $C$ ", "N" and " $U$ " are clearly reconstructed. The corresponding theoretical expectations are shown in Fig. $3 g-i$. The experimental results meet the theoretical expectations quite well. The intensity nonuniformity of the letters is mainly caused by the inhomogeneity of the $\mathrm{THz}$ source. There is still some noise induced by the low signal to noise ratio and the defects of the optical elements.

The photo-generated carriers primarily modulate the transmission of the $\mathrm{THz}$ radiation, so the proposed STM is nearly a pure amplitude modulator. However, the phase distribution of the $\mathrm{THz}$ wave can also be adjusted by using the THz CGH. Here, the vortex beams, which are useful in quantum entanglement and hence enable opportunities in telecommunications $s^{25,26}$, are generated. The complex amplitude of the vortex beams are defined as:

$$
U_{T H z}(r, \phi)=A_{0} \exp \left(\frac{-r^{2}}{\omega_{0}^{2}}\right) \exp (i l \phi),
$$

where $r, \phi$ are the polar coordinates, $A_{0}$ is a constant, $\omega_{0}$ is the waist radius, $l$ is the topological charge of the optical vortex, which is related to the quantum number of the orbital angular momentum. Three THz vortex CGHs with $l=1, l=2$ and $l=3$ are shown in Fig. $4 \mathrm{a}-\mathrm{c}$, respectively. The corresponding intensity distributions captured by the $\mathrm{THz}$ focal plane imaging system are shown in Fig. $4 \mathrm{~d}-\mathrm{f}$, and the phase distributions are shown in Fig. $4 \mathrm{~g}-\mathrm{i}$, respectively. From Fig. $4 \mathrm{~d}-\mathrm{f}$, it can be seen that the intensity distributions of the $\mathrm{THz}$ vortices are typical doughnut shapes, and the size of the hollow part enlarges with the increasing quantum number of the orbital angular momentum. The phase distributions show that the total phase shift around the center are $2 \pi, 4 \pi$ and $6 \pi$ for $l=1$, $l=2$ and $l=3$, respectively. The theoretical phase distributions are also presented in Fig. 4j-1, and good matching between the theoretical and experimental results can be observed.

The modulation rate of this STM is limited by the optical SLM which is 60 frames per second. Considering the recombination time (about $10 \mu \mathrm{s}$ ) of the photo-excited carries ${ }^{27}$, the modulation rate can arrive $10^{5}$ frames per second if a suitable optical projector is used. Limited by the output power of our femtosecond laser source (about $800 \mathrm{~mW}$ ) and the high loss of the optical SLM, the highest intensity 
a

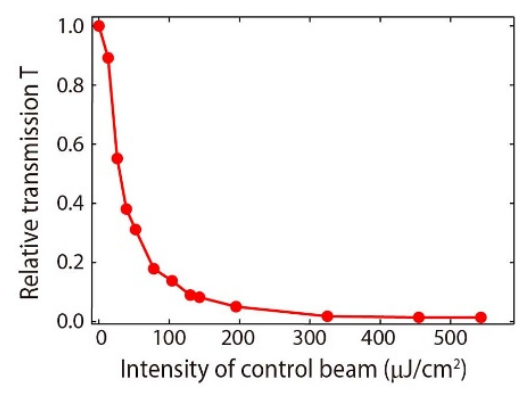

b

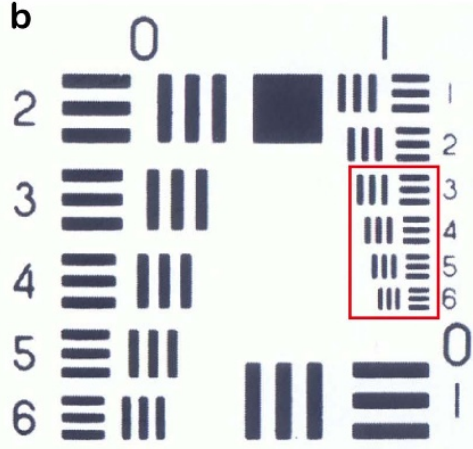

c

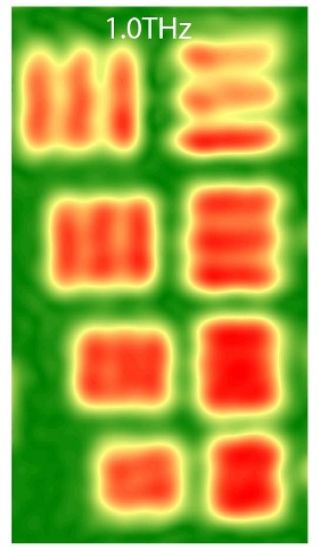

d

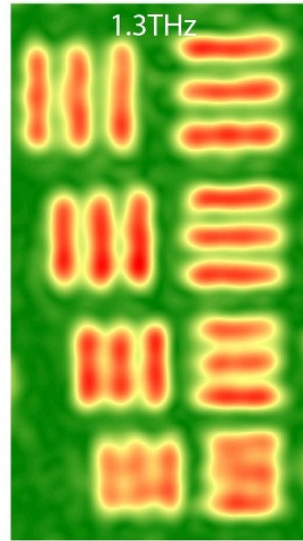

e

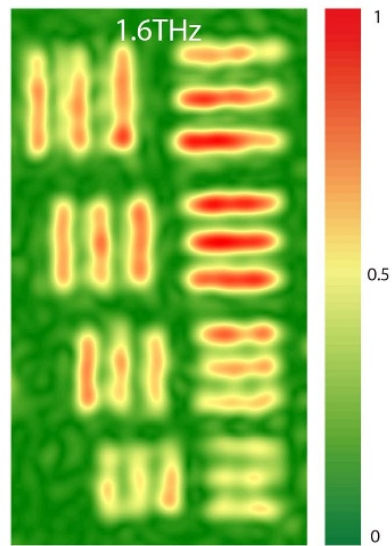

Figure $2 \mid$ Performances of the STM. (a), Transmission of the THz wave through a silicon wafer with respect to the pump intensity. The modulation depth can reach $98.6 \%$ when the pump intensity is $543.2 \mu \mathrm{J} / \mathrm{cm}^{2}$. The transmission exhibits a good linearity with the pump intensity within the range of 0 to $100 \mu \mathrm{J} / \mathrm{cm}^{2}$. (b), Photograph of a 1951 USAF $1 \mathrm{X}$ resolution test chart. (c-e), THz images of the region indicated by the red box in (b) at $1.0 \mathrm{THz}$, $1.3 \mathrm{THz}$, and $1.6 \mathrm{THz}$. The results indicate that the STM can achieve resolution better than $140 \mu \mathrm{m}$.

of the control beam is only $16.4 \mu \mathrm{J} / \mathrm{cm}^{2}$, the modulation depth is only $19.9 \%$, which leads to a low diffraction efficiency about $0.2 \%$. Even under this condition, the good wave front can also be constructed by using this novel method.

An all-optical controllable STM is proposed to dynamically modulate the $\mathrm{THz}$ wave front based on photo-induced carriers. This STM is structure free, high resolution and broadband. Two paradigms of generating $\mathrm{THz}$ special intensity patterns and vortices are exhibited, which verifies the effectiveness, practicability and validity of this STM. It is expected that this novel technology can provide potential applications in the $\mathrm{THz}$ imaging and communication systems.

\section{Methods}

Measurement method. A 100 fs laser pulse with an $800 \mathrm{~nm}$ central wavelength is split into three beams which are the pump beam, probe beam and control beam for generating, detecting and modulating the $\mathrm{THz}$ radiation. The pump beam with a $490 \mathrm{~mW}$ average power firstly passes through a concave lens with a $25 \mathrm{~mm}$ focal length and illuminates a $<110>\mathrm{ZnTe}$ crystal with a $3 \mathrm{~mm}$ thickness. The THz wave, which is excited by the optical rectification in the ZnTe, is collimated by a metallic parabolic mirror (PM) with a $150 \mathrm{~mm}$ focal length. The THz wave with a $42 \mathrm{~mm}$ diameter and a 37 degree incident angle is reflected onto the surface of a silicon wafer by a metallic mirror. The probe beam with a $10 \mathrm{~mW}$ average power is expanded by a lens combination. Then, the probe beam with a $15 \mathrm{~mm}$ diameter is reflected onto the detection crystal (another $<110>$ ZnTe with a $3 \mathrm{~mm}$ thickness) by a 50/50 beam splitter (BS). The reflected probe beam with the $\mathrm{THz}$ information impinges into the imaging module, which consists of two lenses, a quarter wave plate (QWP), a Wollaston prism (PBS), and a CY-DB1300A CCD camera. The imaging module operates the balanced electro-optic detection and the dynamics subtraction technique to extract the $\mathrm{THz}$ information ${ }^{24}$. The control beam with a $300 \mathrm{~mW}$ average power is expanded by a lens combination. The control beam with a $42 \mathrm{~mm}$ diameter

illuminates a Holoeye LC2002 spatial light modulator to carry the pattern of a $\mathrm{THz}$ computer generated hologram $(\mathrm{CGH})$. Then, the $\mathrm{THz} \mathrm{CGH}$ pattern is projected onto the silicon wafer by a convex lens to generate the corresponding photo-induced carrier distribution. After transmitting through the silicon wafer, the diffracted $\mathrm{THz}$

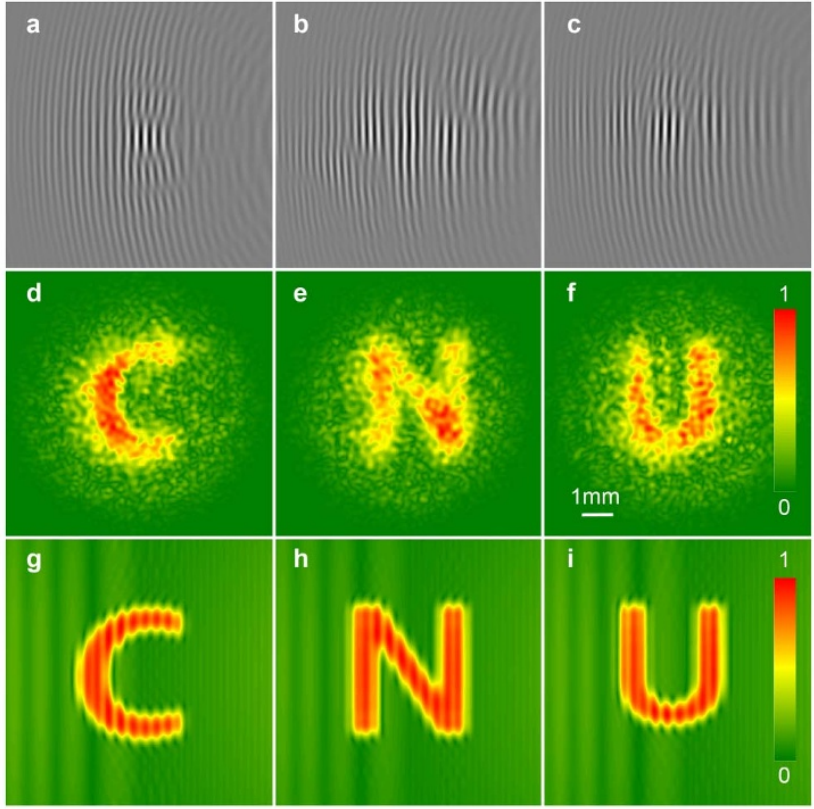

Figure $3 \mid$ Special field distributions generated with the STM. (a-c), Offaxis $\mathrm{THz}$ computer-generated holograms for letters $\mathrm{C}, \mathrm{N}$ and $\mathrm{U}$, respectively. The size of each hologram projected on the silicon wafer is $25.6 \mathrm{~mm} \times 18.2 \mathrm{~mm}$. $(\mathrm{d}-\mathrm{f}), \mathrm{THz}$ intensity distributions on the observation plane. The size of each image is $4 \mathrm{~mm} \times 6 \mathrm{~mm}$. (g-i), Theoretical expectations of the $\mathrm{THz}$ intensity distributions on the preset plane for letters $\mathrm{C}, \mathrm{N}$ and $\mathrm{U}$, respectively. 


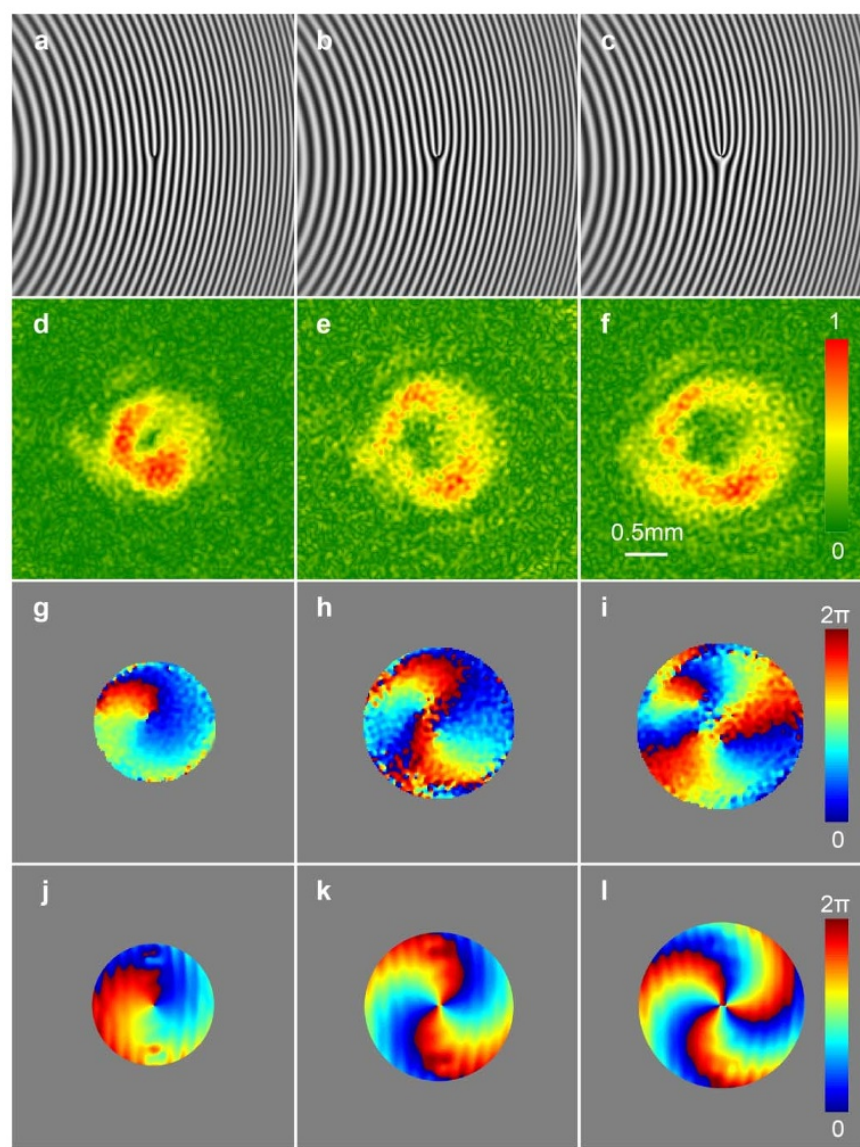

Figure $4 \mid$ Vortex beams generated with the STM. (a-c), Off-axis terahertz computer-generated holograms for the $\mathrm{THz}$ vortices with orbital angular momentum quantum number $l=1, l=2$ and $l=3$, respectively. (d-f), Intensity distributions of the $\mathrm{THz}$ vortex beams on the observation plane for $l=1, l=2$ and $l=3$. (g-i), Corresponding phase distributions. ( $\mathrm{j}-1)$, Theoretical expectations of the phase distribution of the THz vortex beams on the preset plane for $l=1, l=2$ and $l=3$, respectively.

wave will form the preset field distribution on the detection plane. To acquire the $\mathrm{THz}$ information, the CCD camera is synchronized with the chopper inserted into the control beam. The control beam arrive at the silicon wafer 10 ps earlier than the $\mathrm{THz}$ wave to ensure that the $\mathrm{THz}$ wave can be modulated by the photo-induced carriers. By adjusting the optical path difference between the $\mathrm{THz}$ wave and the probe beam, $64 \mathrm{THz}$ temporal images at different scan points are acquired. At each scan point, 100 frames are averaged to enhance the signal to noise ratio. The corresponding scanning time window is $8.5 \mathrm{ps}$.

1. Tonouchi, M. Cutting-edge terahertz technology. Nature Photon. 1, 97-105 (2007).

2. Williams, G. P. Filling the terahertz gap-high power sources and applications. Rep. Prog. Phys. 69, 301-326 (2006)

3. Siegel, P. Terahertz technology. IEEE Trans. Microw. Theory Tech. 50, 910-927 (2002).

4. Efron, U. Spatial light modulator technology: Materials, devices and applications (CRC Cleveland/Boca Raton, 1995).

5. Köhler, R. et al. Terahertz semiconductor-heterostructure laser. Nature 417 , 156-159 (2002).

6. Willians, B. S. Terahertz quantum-cascade lasers. Nature Photon. 1, 517-525 (2007).
7. Kawase, K. et al. Tunable terahertz-wave generation from DAST crystal by dual signal-wave parametric oscillation of periodically poled lithium niobate. Opt. Lett. 25, 1714-1716 (2000)

8. Exter, M. V., Fattinger, C. \& Grischkowsky, D. Terahertz time-domain spectroscopy of water vapor. Opt. Lett. 14, 1128-1130 (1989).

9. Chen, H. T. et al. Active terahertz metamaterial devices. Nature 444, 597-600 (2006).

10. Landy, N. I. et al. Perfect metamaterial absorber. Phys. Rev. Lett. 100, 207402 (2008).

11. Ren, L. et al. Broadband terahertz polarizers with ideal performance based on aligned carbon nanotube stacks. Nano Lett. 12, 787-790 (2012).

12. Sensale-Rodriguez, B. et al. Extraordinary control of terahertz beam reflectance in graphene electro-absorption modulators. Nano Lett. 12, 4518-4522 (2012).

13. Sensale-Rodriguez, B. et al. Broadband graphene terahertz modulators enabled by intraband transitions. Nature communications 3, 780 (2012).

14. Grady, N. K. et al. Terahertz metamaterials for linear polarization conversion and anomalous refraction. Science 340, 1304-1307 (2013).

15. Rizza, C. et al. Terahertz active spatial filtering through optically tunable hyperbolic metamaterials. Opt. Lett. 37, 3345-3347 (2012).

16. Chen, H. T. et al. A metamaterial solid-state terahertz phase modulator. Nature Photon. 3, 141-151 (2009).

17. Gu, J. et al. Active control of electromagnetically induced transparency analogue in terahertz metamaterials. Nature Commun. 3, 1151 (2012).

18. Smith, D. R., Pendry, J. B. \& Wiltshire, M. C. K. Metamaterials and negative refractive index. Science 395, 788-792 (2004).

19. Yu, N. et al. Light propagation with phase discontinuities: Generalized laws of reflection and refraction. Science 334, 333-337 (2011).

20. Hu, D. et al. Ultrathin terahertz planar elements. Adv. Opt. Mater. 1, 186-191 (2013).

21. Ferguson, B. \& Zhang, X. C. Materials for terahertz science and technology. Nature Mater. 1, 26-33 (2002).

22. Chan, W. L. et al. A spatial light modulator for terahertz beams. Appl. Phys. Lett. 94, 213511 (2009).

23. Wang, X. K. et al. Terahertz real-time imaging with balanced electro-optics detection. Opt. Commun. 283, 4626-4632 (2010).

24. Wang, X. K. et al. Terahertz polarization real-time imaging based on balanced electro-optic detection. J. Opt. Soc. Am. A 27, 2387-2393 (2010).

25. Fickler, R. et al. Quantum entanglement of high angular momenta. Science 338, 640-643 (2012).

26. Wang, J. et al. Terabit free-space data transmission employing orbital angular momentum multiplexing. Nature Photonics 6, 488-496 (2012)

27. Schroder, D. K. Carrier lifetimes in silicon. Electron Devices, IEEE Transactions on 44, 160-170 (1997).

\section{Acknowledgments}

This work was supported by the 973 Program of China (No. 2013CBA01702), the National Natural Science Foundation of China (No. 11204188, 61205097, 91233202, 11374216, and 11174211), the National High Technology Research and Development Program of China (No. 2012AA101608-6), the Beijing Natural Science Foundation (No. KZ201110028035 and 1132011), the Program for New Century Excellent Talents in University (NCET-12-0607), and the CAEP THz Science and Technology Foundation.

\section{Author contributions}

Z. Xie and X. Wang conceived and designed the experiments. J. Ye, S. Feng and W. Sun designed the holograms. T. Akalin and Y. Zhang guided the theoretical and experimental work. All authors discussed the results and co-wrote the manuscript.

\section{Additional information}

Competing financial interests: The authors declare no competing financial interests.

How to cite this article: Xie, Z.W. et al. Spatial Terahertz Modulator. Sci. Rep. 3, 3347; DOI:10.1038/srep03347 (2013).

(c) (i) (2) This work is licensed under a Creative Commons Attribution-

BY NC SA NonCommercial-ShareAlike 3.0 Unported license. To view a copy of this license, visit http://creativecommons.org/licenses/by-nc-sa/3.0 